Interesting Images

\title{
Carcinoma with Triphasic Differentiation Arising from Inverted Papilloma in Sinonasal Sinus: A Rare Case with Molecular Characterization
}

\author{
So-Woon Kim and Kiyong $\mathrm{Na} *$ (1) \\ Department of Pathology, Kyung Hee University Hospital, College of Medicine, Kyung Hee University, \\ Seoul 02447, Korea; sowoonkim86@gmail.com \\ * Correspondence: raripapa@gmail.com; Tel.: +82-29588740
}

check for updates

Citation: Kim, S.-W.; Na, K.

Carcinoma with Triphasic

Differentiation Arising from Inverted Papilloma in Sinonasal Sinus: A Rare Case with Molecular Characterization. Diagnostics 2021, 11, 1827. https://

doi.org/10.3390/diagnostics11101827

Academic Editor: Alessio Imperiale

Received: 17 August 2021

Accepted: 30 September 2021

Published: 3 October 2021

Publisher's Note: MDPI stays neutral with regard to jurisdictional claims in published maps and institutional affiliations.

Copyright: (c) 2021 by the authors. Licensee MDPI, Basel, Switzerland. This article is an open access article distributed under the terms and conditions of the Creative Commons Attribution (CC BY) license (https:/ / creativecommons.org/licenses/by/ $4.0 /)$.

\begin{abstract}
Small cell neuroendocrine carcinoma (SNEC) is a rare subset of tumors in the sinonasal sinus. Combined tumors are exceedingly rare. Here, we describe a 65-year-old male with a mixed tumor of SNEC and sarcomatoid carcinoma arising in an inverted papilloma, containing squamous cell carcinoma in situ (SqCCis) in the sinonasal sinus. We evaluated the molecular characteristics of the two separate carcinoma components using next-generation sequencing. The patient presented with a nasal obstruction. Computed tomography showed a mass infiltrating the right ethmoid and maxillary sinuses. An excisional biopsy was performed. The tumor was found to have three morphologically distinct components. The first was SqCCis arising in an inverted papilloma, which was positive for cytokeratin and P40. The second consisted of nests of densely packed small round cells representing SNEC-positive neuroendocrine markers. The third was a solid sheet of anaplastic spindle cell proliferation, which was negative for the above markers. Oncogenic mutations such as FBXW7, TP53, and EGFR were detected in both SNEC and sarcomatoid carcinoma, and MYCL amplification was observed only in the SNEC component. This case highlights an extremely rare presentation of combined SNEC and sarcomatoid carcinoma arising from an inverted papilloma in the sinonasal sinus.
\end{abstract}

Keywords: small cell neuroendocrine carcinoma; sarcomatoid carcinoma; squamous cell carcinoma; sinonasal sinus; composite tumor

Tumors of the sinonasal tract are rare, representing approximately $3 \%$ of head and neck malignancies and approximately $0.3 \%$ of all malignant tumors [1]. The most frequent histologic subtype is squamous cell carcinoma (SqCC), followed by adenocarcinoma, melanoma, and olfactory neuroblastoma [1,2]. Small cell neuroendocrine carcinoma (SNEC) is a rare tumor in the head and neck region that occurs most frequently in the larynx, while sinonasal sinuses are exceedingly rare primary sites [3].

Only a small case series and case reports have been published regarding primary sinonasal tract SNEC [3]. In addition, only a few examples of combined tumor have been reported in the sinonasal tract. These consisted mainly of a combination of adenocarcinoma and neuroendocrine carcinoma, while cases of combined small cell and SqCC appear to be very rare [1,4]. Furthermore, the collision of three components (neuroendocrine cells, sarcomatous cells, and squamous cells) in a solid tumor is extremely rare, and no reports of combined tumors with these triphasic features have been reported in the literature [2].

Here, we describe the clinicopathological, immunohistochemical and molecular features of primary combined SNEC and sarcomatoid carcinoma arising from an inverted papilloma containing squamous cell carcinoma in situ (SqCCis) in the sinonasal sinus.

Next, we performed next-generation sequencing on each SNEC and the sarcomatoid carcinoma component to analyze the molecular heterogeneity of the tumor. As a result, oncogenic mutations, such as FBXW7 (c.1972C>T, p. R658*), TP53 (c.740A>T, p. N247I), 
and EGFR (c.2319_2320insAACCCCCAC, p. D770_N771insNPH) were detected in both the SNEC and sarcomatoid carcinoma components. Of note, MYCL amplification was observed only in the SNEC component, a frequent oncogenic event in SNEC, supporting our diagnosis. [5]. FBXW7 is well known to be highly frequently mutated in SqCC, and a loss of TP53 and mutation of FBXW7 in SqCC are known to lead to resistance to standard chemotherapy [6,7]. Therefore, it seems that this heterogeneous tumor arises from a single SqCC component and generates SNEC through further molecular alterations. The final stage was T4bN0M1.

After the biopsy, the patient underwent radiation therapy of $70.2 \mathrm{~Gy}$. However, six months after surgical resection and radiation therapy, follow-up brain CT showed new round masses abutting the superior falx cerebri, left parietal lobe, and both frontal lobes (Figure 1D). The patient died later that month.

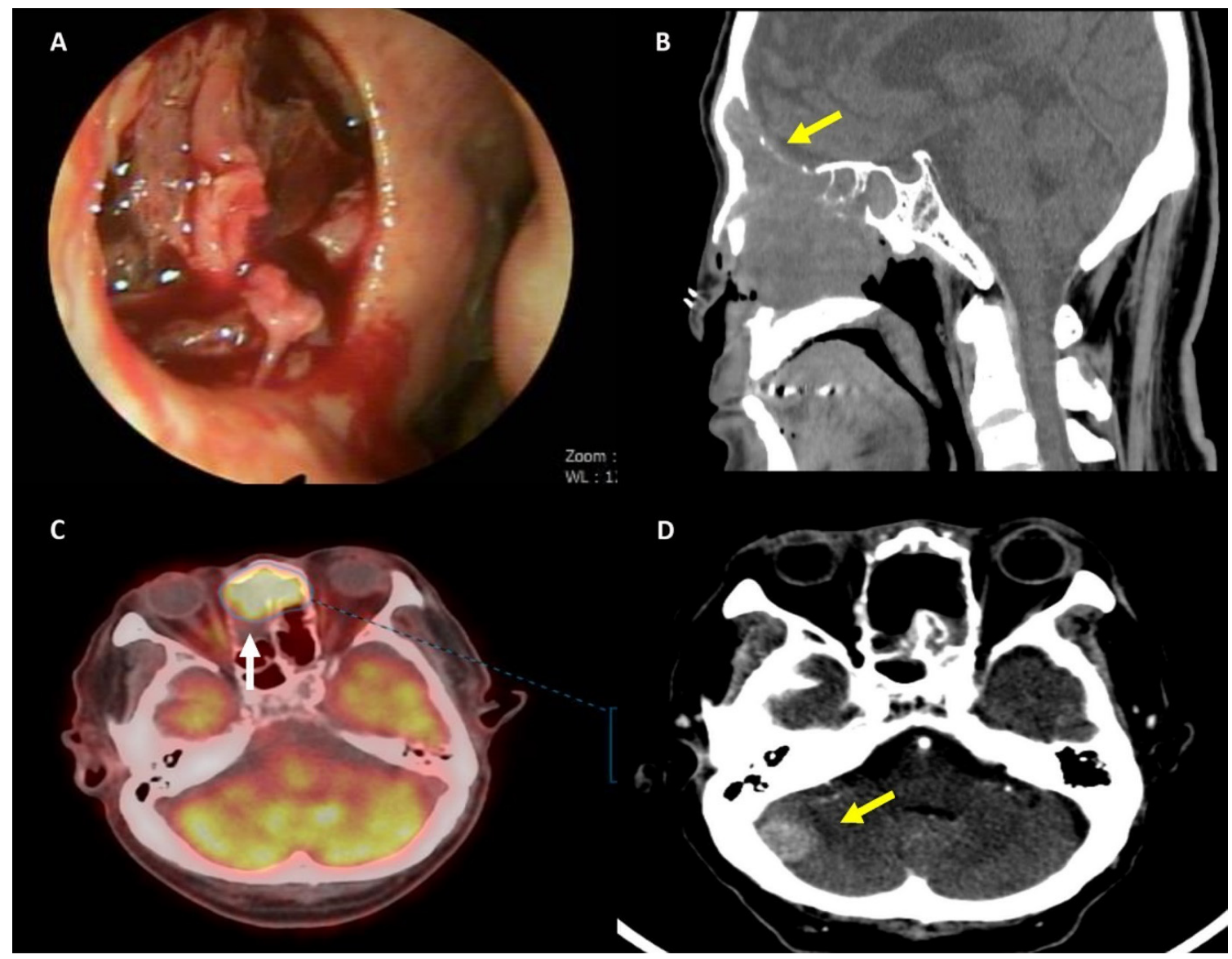

Figure 1. A 65-year-old male was referred to the department of otorhinolaryngology with epistaxis and right nasal obstruction for two months. His medical history revealed diabetes mellitus, hypertension, and pulmonary tuberculosis. (A) Physical examination demonstrated an easy-bleeding tumor in the right nasal cavity and bloody crust with an ulcerative lesion at the anterior septum; (B) The initial computed tomography (CT) showed a $4.5 \mathrm{~cm} \times 3.4 \mathrm{~cm} \times 4.8 \mathrm{~cm}$ soft tissue lesion infiltrating the right ethmoid and maxillary sinuses with destruction at the frontal sinus posterior wall and cribriform plate. Magnetic resonance imaging (MRI) showed a residual mass with destruction in the posterior wall of the frontal sinus, cribriform plate, and dura invasion with suspicious brain parenchyma (right frontal lobe) invasion; (C,D) Fluorodeoxyglucose positron emission tomography (FDG-PET) showed a residual hypermetabolic mass involving the superior portion of the nasal cavity and the anterior portion of the ethmoid sinus, with a maximum standardized uptake value (SUVmax) of 8.5. Clinical differential diagnoses included inverted papilloma with malignant transformation, lymphoma, and olfactory neuroblastoma. To confirm the diagnosis, an excisional biopsy was conducted.

This case highlights an extremely rare presentation of primary combined SNEC and sarcomatoid carcinoma arising from an inverted papilloma in the sinonasal sinus. To the 
best of our knowledge, there have been no cases of combined SNEC and sarcomatoid carcinoma diagnosed in the sinonasal sinus reported in the literature. Since it can affect the treatment plan of chemotherapy or radiation therapy depending on the components constituting the tumor, the need for accurate pathological diagnosis and technology is required. Our findings can help pathologists and clinicians make accurate histological diagnoses of combined SNEC and sarcomatoid carcinoma and plan an adequate treatment strategy for this rare tumor.

The stains used were hematoxylin and eosin stain (Figures 2 and 3) and the polymer method (Figure 3). The original magnifications were $40 \times$ in Figure 2, 400 $\times$ in the inset of Figure 2, and 200× in Figure 3.

\section{A SqCC in situ arising in inverted papilloma B Neuroendocrine carcinoma}

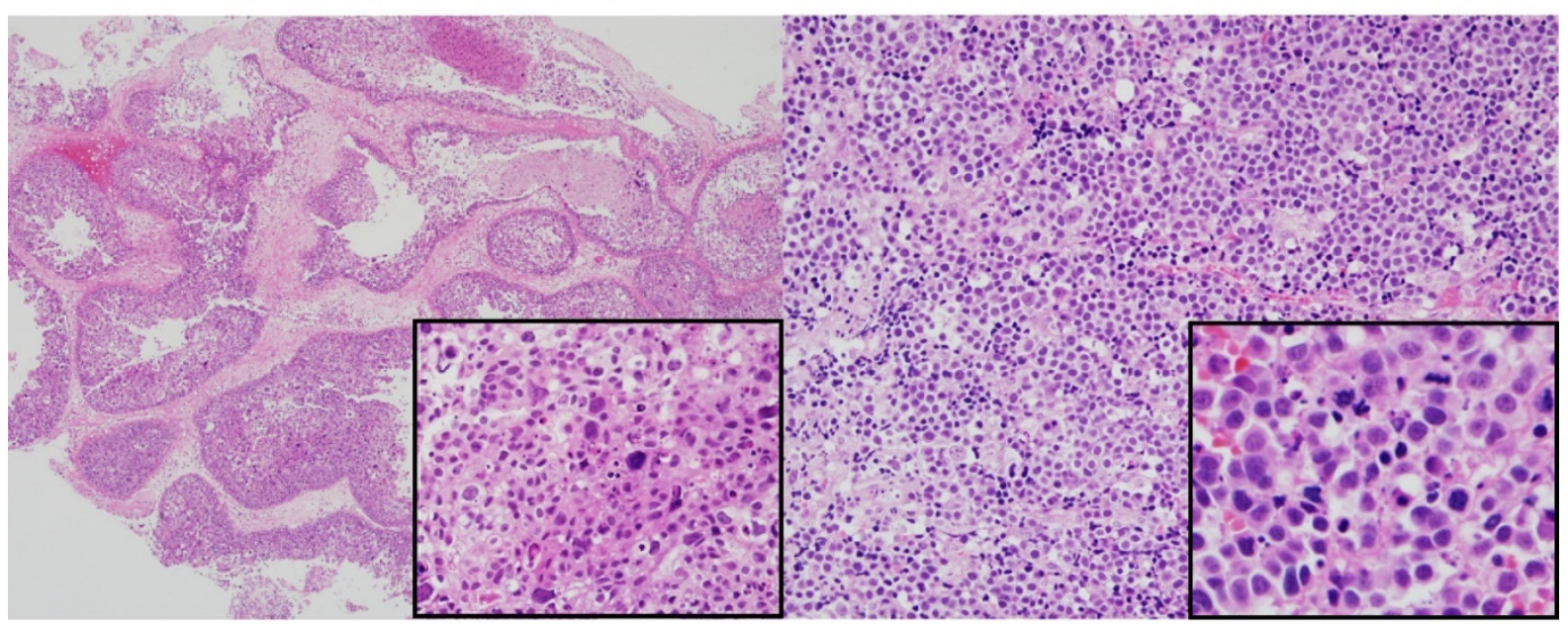

C Sarcomatoid carcinoma

D Mixed area

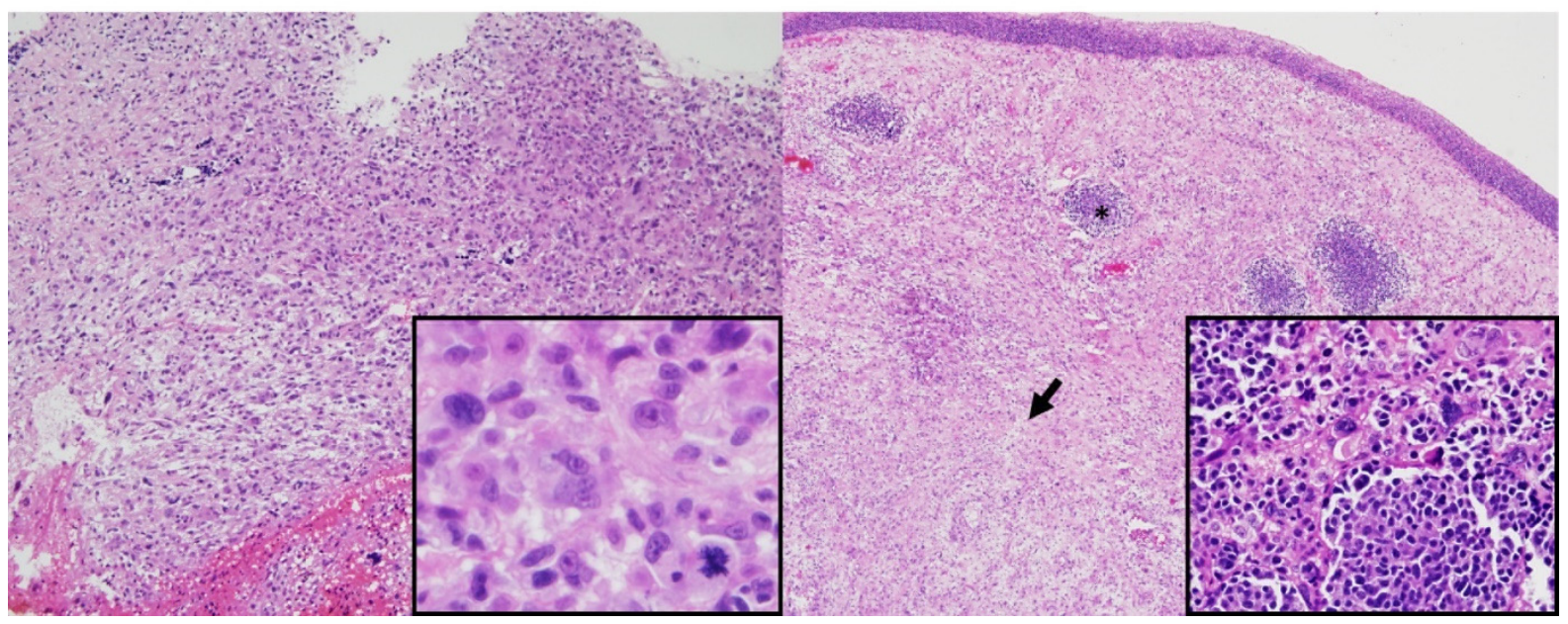

Figure 2. Endoscopic excisional biopsy of the right nasal cavity mass was performed. The tumor was found to consist of three morphologically distinct components in the resected specimen. These components were partly clearly separated and partly strictly mixed. (A) The first component was SqCCis arising in inverted papilloma, which represented approximately $20 \%$ of the lesion. (B) The second component comprised of nests of closely packed, small round to atypical oval cells, with hyperchromatic nuclei, coarse chromatin, and scant pale eosinophilic cytoplasm. The tumor had karyorrhectic debris and mitotic activity. (C) This represented about $40 \%$ of the lesion. The third component consisted of a solid sheet of anaplastic spindle cells proliferation with prominent mitosis and multinucleated giant cells, which represented the remaining $40 \%$ of the lesion. (D) Of note, the focal area showed a tumor with admixed small round cell nests and anaplastic spindle cell elements overlying SqCCis. Asterisk, small round cell nest; arrow, spindle cell proliferation; right upper, squamous cell carcinoma in situ. The original magnifications were $40 \times$ and $400 \times$ in the inset 


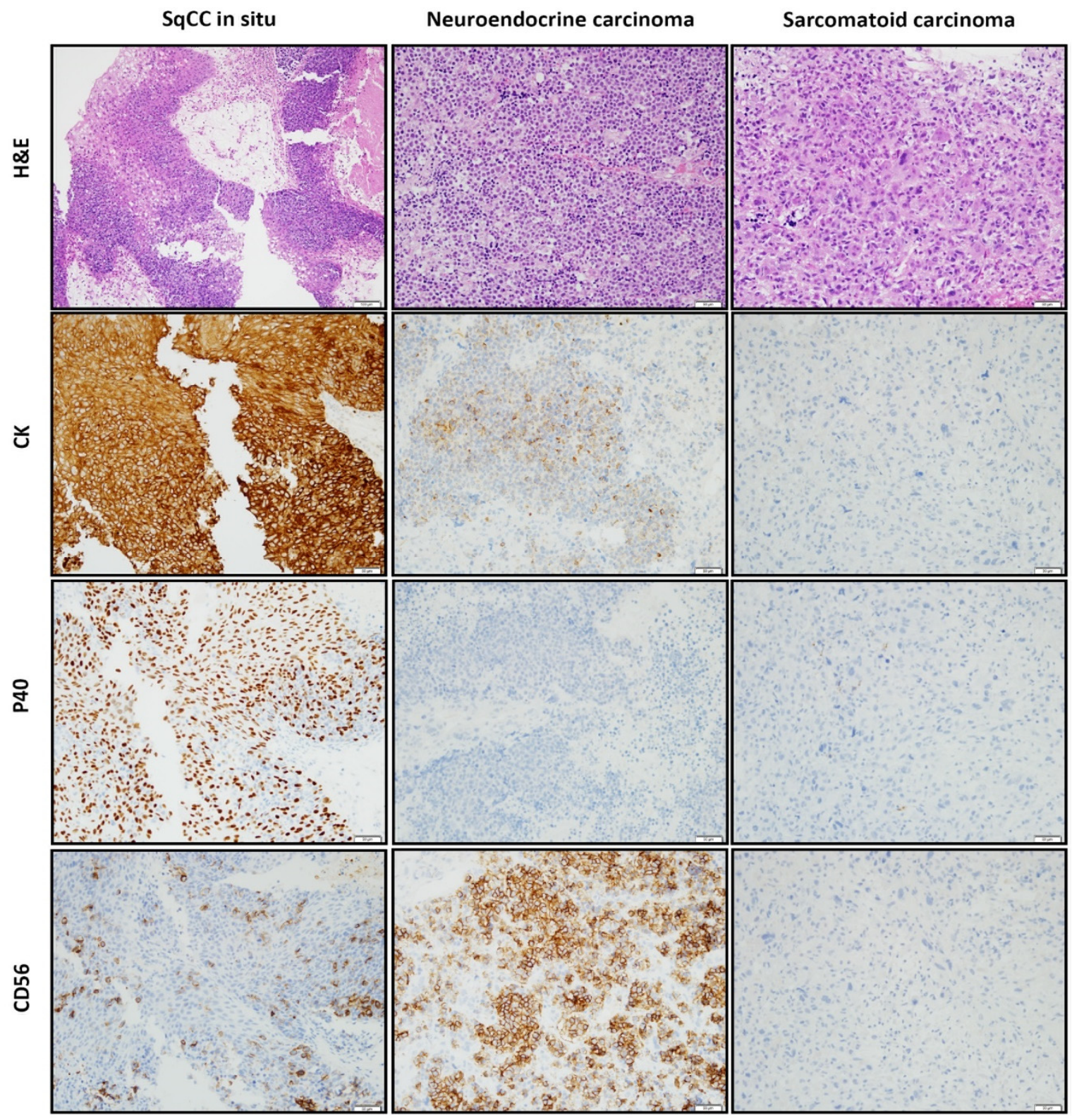

Figure 3. To characterize each component, an immunohistochemical examination was performed. The results of the immunohistochemical studies are illustrated in Figure 3. (Left column) The three components clearly showed differences in their immunoprofiles. SqCCis was diffusely cytoplasmic positive for pan-cytokeratin (CK) and nuclear positive for P40, but negative for the neuroendocrine markers CD56, synaptophysin, and chromogranin. Conversely, the nests of atypical small cells were positive for $\mathrm{CK}$ with a perinuclear dot pattern and diffusely cytoplasmic positive for the neuroendocrine markers CD56, synaptophysin, and chromogranin. (Middle column) However, the nests were negative for P40. (Right column) The anaplastic spindle cell lesion was negative for all markers. Based on the histologic features and the results of the immunohistochemical studies, a diagnosis of combined SNEC and sarcomatoid carcinoma arising in an inverted papilloma was rendered. It is important to distinguish whether a spindle cell lesion is another sarcoma or a spindle cell variant of SqCC (sarcomatoid carcinoma). A diagnosis of sarcomatoid carcinoma can be considered if the existing conventional squamous cell carcinoma is mixed with the spindle cell lesion or if the SqCCis is present together [8,9]. In our case, because the spindle cell component coexisted with SqCCis, we diagnosed this component as sarcomatoid carcinoma rather than another sarcoma. The original magnifications were $200 \times$.

Author Contributions: Conceptualization, S.-W.K. and K.N.; formal analysis: S.-W.K.; investigation, K.N.; writing —original draft preparation; S.-W.K. and K.N.; writing—review and editing, S.-W.K. 
and K.N.; visualization, S.-W.K.; supervision, S.-W.K. and K.N.; project administration, S.-W.K. Both authors read and agreed to the published version of the manuscript.

Funding: This research was funded by the Basic Science Research Program through the National Research Foundation of Korea (NRF), funded by the Ministry of Science, ICT, and Future Planning (NRF-2020R1G1A1003692).

Institutional Review Board Statement: The procedures were conducted in accordance with the Declaration of Helsinki. This study (project identification code: 2020-03-081, date of approval: 21 March 2020) was reviewed and approved by the Institutional Review Board of Kyung Hee University Hospital (Seoul, Korea).

Informed Consent Statement: All subjects provided informed consent for inclusion before they participated in the study.

Data Availability Statement: The data presented in this study are available upon request from the corresponding author. The data are not publicly available because of privacy and ethical restrictions.

Conflicts of Interest: The authors declare no conflict of interest regarding this study.

\section{References}

1. $\mathrm{Wu}, \mathrm{S.H}$.; Zhang, B.Z.; Han, L. Collision tumor of squamous cell carcinoma and neuroendocrine carcinoma in the head and neck: A case report. World J. Clin. Cases 2020, 8, 2610-2616. [CrossRef] [PubMed]

2. Huang, S.F.; Chuang, W.Y.; Cheng, S.D.; Hsin, L.J.; Lee, L.Y.; Kao, H.K. A colliding maxillary sinus cancer of adenosquamous carcinoma and small cell neuroendocrine carcinoma-a case report with EGFR copy number analysis. World J. Surg. Oncol. 2010, 8, 92. [CrossRef] [PubMed]

3. Bell, D. Sinonasal Neuroendocrine Neoplasms: Current Challenges and Advances in Diagnosis and Treatment, with a Focus on Olfactory Neuroblastoma. Head Neck Pathol. 2018, 12, 22-30. [CrossRef] [PubMed]

4. Franchi, A.; Rocchetta, D.; Palomba, A.; Degli Innocenti, D.R.; Castiglione, F.; Spinelli, G. Primary combined neuroendocrine and squamous cell carcinoma of the maxillary sinus: Report of a case with immunohistochemical and molecular characterization. Head Neck Pathol. 2015, 9, 107-113. [CrossRef] [PubMed]

5. Mollaoglu, G.; Guthrie, M.R.; Böhm, S.; Brägelmann, J.; Can, I.; Ballieu, P.M.; Marx, A.; George, J.; Heinen, C.; Chalishazar, M.D.; et al. MYC Drives Progression of Small Cell Lung Cancer to a Variant Neuroendocrine Subtype with Vulnerability to Aurora Kinase Inhibition. Cancer Cell 2017, 31, 270-285. [CrossRef]

6. Yeh, C.-H.; Bellon, M.; Nicot, C. FBXW7: A critical tumor suppressor of human cancers. Mol. Cancer 2018, 17, 115. [CrossRef]

7. Sailo, B.L.; Banik, K.; Girisa, S.; Bordoloi, D.; Fan, L.; Halim, C.E.; Wang, H.; Kumar, A.P.; Zheng, D.; Mao, X.; et al. FBXW7 in Cancer: What Has Been Unraveled Thus Far? Cancers 2019, 11, 246. [CrossRef]

8. Mahajan, A.; Mohanty, S.; Ghosh, S.; Urs, A.B.; Khurana, N.; Gupta, S. Sarcomatoid Carcinoma of the Oral Cavity: A Diagnostic Dilemma. Case Rep. Dent. 2017, 2017, 7495695. [CrossRef] [PubMed]

9. Choi, H.-R.; Sturgis, E.M.; Rosenthal, D.I.; Luna, M.A.; Batsakis, J.G.; El-Naggar, A.K. Sarcomatoid Carcinoma of the Head and Neck: Molecular Evidence for Evolution and Progression From Conventional Squamous Cell Carcinomas. Am. J. Surg. Pathol. 2003, 27, 1216-1220. [CrossRef] [PubMed] 